\section{DERNIÈRE HEURE}

\section{Identification de mutations activatrices de gP 130 dans la tumorigenèse hépatique}

Jessica Zucman-Rossi, Mohamed Amessou, Paulette Bioulac-Sage, Sandra Rebouissou
J. Zucman-Rossi, M. Amessou,

S. Rebouissou : Inserm U674,

Équipe Génétique des tumeurs hépatiques, Université Paris Diderot,

27, rue Juliette Dodu, 75010 Paris, France. zucman@cephb.fr

P. Bioulac-Sage : Inserm U889,

Université Bordeaux 2,

Service d'anatomie pathologique, Hôpital Pellegrin, CHU de Bordeaux, place Amélie Raba-Léon, 33076 Bordeaux Cedex, France.

\section{Les adénomes hépatocellulaires}

Les adénomes hépatocellulaires sont des tumeurs bénignes du foie qui se développent le plus souvent chez la femme jeune après la prise de contraceptifs oraux et en l'absence de pathologie chronique du foie. Récemment, nous avons décrit un type particulier d'adénomes caractérisés par la présence d'un infiltrat inflammatoire au sein de la tumeur [1]. Ces adénomes sont fréquemment télangiectasiques, c'est-àdire qu'ils présentent des remaniements vasculaires à type de dilatation sinusoïdale et leur développement est souvent associé à une surcharge pondérale et à la prise d'alcool [2]. Grâce à une étude du transcriptome de ces tumeurs, nous avons pu montrer une surexpression de protéines de l'inflammation dans les cellules tumorales [2]. La voie de l'interleukine-6 (IL-6), mais aussi les voies des interférons de type 1 et 2 sont activées dans ces tumeurs, aboutissant notamment à la surexpression de l'ensemble des protéines de la phase aiguë de l'inflammation [3]. Ainsi, un syndrome inflammatoire périphérique a souvent été identifié chez ces patients résultant de l'augmentation de la concentration plasmatique de protéines inflammatoires comme CRP (C reactive protein) et SAA (serum amyloid protein A) sécrétées par les cellules tumorales [4].

\section{Identification des mutations de gp130} dans les adénomes inflammatoires Nous avons montré par immunohistochimie que l'expression des protéines inflammatoires était restreinte aux cellules tumo- rales de l'adénome, sans renforcement de l'immunomarquage à proximité des infiltrats inflammatoires dans la tumeur, ni expression dans le foie non tumoral. Cette observation nous a conduit à émettre l'hypothèse selon laquelle l'activation de la voie inflammatoire pouvait être le résultat d'une altération génétique présente dans les cellules tumorales, l'infiltrat inflammatoire (essentiellement lymphocytaire polymorphe) étant secondairement recruté par les cellules tumorales. Cette hypothèse nous a amené à chercher des mutations des gènes codant les différents acteurs de la voie de signalisation de I'IL-6 par séquençage de l'ADN tumoral. Cette stratégie nous a permis d'identifier des mutations dans le gène IL6ST (IL-6 signal transducer) codant la gpl30 qui est le co-récepteur de I'IL-6 mais aussi d'autres cytokines comme l'IL-11, I'IL-27, le LIF (leukemia inhibitory factor), I'OSM (oncostatine), le CNTF (ciliary neurotrophic factor) et la CT-1 (cardiotrophine-1) [5-7]. Toutes les mutations identifiées sont monoalléliques et d'origine somatique; elles ont été mises en évidence dans 26 des 43 cas d'adénomes inflammatoires analysés. Dans la plupart des cas, ces mutations conduisent à la délétion en phase de quelques acides aminés localisés au niveau du site de fixation physiologique de l'IL-6 sur la gpl30 (Figure I).

\section{Les mutants gp130 activent la voie} de I'IL-6 indépendamment du ligand Par mutagenèse dirigée, nous avons reproduit 4 délétions différentes dans la séquence codante de gpl30 sous le contrôle d'un promoteur eucaryote. L'analyse de la surexpression des mutants gp 130 dans des cellules hépatocellulaires Hep3B a montré que tous les mutants sont capables d'activer la voie inflammatoire IL6/STAT3 et ce, en l'absence d'IL-6. Cette activité est dépendante de la quantité de mutant exprimé et est diminuée par la surexpression du co-récepteur gp130 sauvage. Ainsi, nous avons montré que ces formes mutées du co-récepteur étaient constitutivement actives en l'absence du ligand physiologique. Par ailleurs, il est bien établi que la transduction du signal IL-6 nécessite la dimérisation de la gp130 consécutive à la formation d'un complexe protéique hexamèrique incluant deux molécules de chaque partenaire, I'IL-6, le récepteur à l'IL-6 (gp80) et la gp130. A cet égard, nous avons montré que le co-récepteur gpl30 muté est capable de s'homodimériser en l'absence d'IL-6 et que cela constitue probablement l'étape clef dans le mécanisme d'activation de la voie IL-6/STAT3 dans les adénomes hépatocellulaires inflammatoires.

La voie gp130 pourrait coopérer avec la voie WNT/ $\beta$-caténine pour la transformation maligne Pour tester une possible interaction entre la voie de I'IL-6 et les autres voies de la carcinogenèse qui sont altérées dans les adénomes hépatocellulaires, nous avons analysé une série de 76 adénomes hépatocellulaires à la recherche des mutations inactivatrices d'HNFl $\alpha$ (hepatocyte 


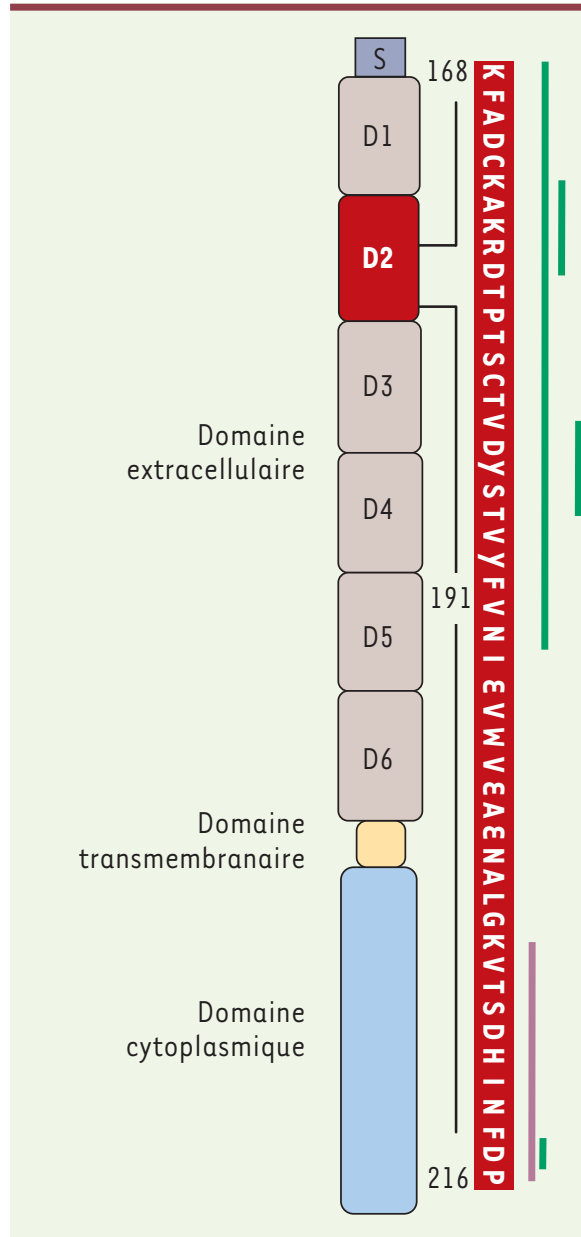

nuclear factor $1 \alpha$ ) [8], activatrices de la $\beta$-caténine [9] et de gp130. Les mutations d'HNFl $\alpha$ et de gpl30 sont mutuellement exclusives car aucune tumeur n'est mutée pour les deux gènes $\left(P<10^{-4}\right)$; au contraire, la moitié des tumeurs exprimant une mutation activatrice de la $\beta$-caténine possèdent aussi une mutation de gp130. Deux de ces adénomes porteurs d'une double mutation se sont transformés en carcinome hépatocellulaire alors qu'aucune transformation maligne n'a été observée dans les adénomes présentant uniquement une mutation de gp130. Par ailleurs, lorsque nous avons recherché les mutations de gpl30 dans une série de 111 carcinomes hépatocellulaires d'étiologies variées et sans histoire connue d'adénome, nous avons identifié deux carcinomes hépatocellulaires avec une mutation activatrice de gp130. De manière surprenante, ces deux cas étaient développés sur foie sain, en l'absence de cirrhose et

\section{REMERCIEMENTS}

Ce travail a été financé par l'Inserm, l'ARC, la Ligue Nationale contre le cancer et la Fondation de France. Il a été effectué en collaboration avec les cliniciens et anatomopathologistes du CHU de Bordeaux et avec Tina Izard à Scripps Research Institute, Floride.

\section{RÉFÉRENCES}

1. Zucman-Rossi J, Jeannot $\varepsilon$, Nhieu JT, et al. Genotype-phenotype correlation in hepatocellular adenoma: new classification and relationship with HCC. Hepatology 2006 ; 43 : 515-24.

2. Bioulac-Sage P, Rebouissou S, Thomas C, et al. Hepatocellular adenoma subtype classification using molecular markers and immunohistochemistry. Hepatology 2007 ; $46: 740-8$.

3. Rebouissou S, Amessou M, Couchy G, et al. Frequent in-frame somatic deletions activate gp 130 in inflammatory hepatocellular tumours. Nature 2008 ; PMID 19020503 (sous presse).

Figure 1. Représentation des différentes mutations somatiques de gp130 identifiées dans les adénomes hépatocellulaires inflammatoires. La structure de la protéine gp130 avec ses différents domaines est indiquée sur la gauche. La séquence en acides aminés d'une partie du domaine D2 est indiquée en rouge; les lignes vertes représentent les acides aminés délétés en phase dans les tumeurs; en violet, un événement de duplication en phase.

une mutation activatrice de la $\beta$-caténine a été identifiée dans chaque tumeur. Ces résultats suggèrent l'existence d'une coopération entre la voie de l'IL-6 et celle de la $\beta$-caténine dans le processus de la transformation maligne.

\section{Perspectives}

Par ce travail, nous avons mis en évidence pour la première fois des mutations activatrices de gpl30 dans des tumeurs humaines, identifiant le co-recepteur de I'IL-6 comme un nouvel oncogène. Ainsi, nous avons établi un nouveau mécanisme associant l'inflammation et la tumorigenèse $[10,11]$. Dans l'avenir, il sera intéressant de tester ces altérations génétiques dans d'autres types de tumeurs, en particulier les tumeurs inflammatoires présentant une activation inexpliquée de STAT3. $\diamond$

\section{Gp130 activating mutations}

in inflammatory adenomas
4. Sa Cunha A, Blanc JF, Lazaro $\varepsilon$, et al. Inflammatory syndrome with liver adenomatosis: the beneficial effects of surgical management. Gut 2007 ; $56: 307-9$.

5. Akira S, Nishio Y, Inoue M, et al. Molecular cloning of APRF, a novel IFN-stimulated gene factor 3 p91related transcription factor involved in the gp 130mediated signaling pathway. Cell 1994; $77: 63-71$.

6. Hibi M, Murakami M, Saito M, et al. Molecular cloning and expression of an IL-6 signal transducer, gp130. Cell $1990 ; 63: 1149-57$.

7. Stahl N, Boulton TG, Farruggella T, et al. Association and activation of Jak-Tyk kinases by CNTF-LIF-OSMIL-6 beta receptor components. Science 1994 ; $263: 92-5$.

8. Bluteau 0, Jeannot $\varepsilon$, Bioulac-Sage P, et al. $\mathrm{Bi}$-allelic inactivation of TCFl in hepatic adenomas. Nat Genet $2002 ; 32$ : 312-5.

9. Chen YW, Jeng YM, Yeh SH, Chen PJ. P53 gene and Wnt signaling in benign neoplasms: beta-catenin mutations in hepatic adenoma but not in focal nodular hyperplasia. Hepatology 2002 ; 36 : 927-35.

10. Grivennikov S, Karin M. Autocrine IL-6 signaling: a key event in tumorigenesis? Cancer Cell 2008; $13: 7-9$.

11. Naugler WE, Sakurai T, Kim S, et al. Gender disparity dependent IL-6 production. Science 2007 ; 317: 121-4.

\section{TIRÉS À PART}

J. Zucman-Rossi in liver cancer due to sex differences in MyD88- 